\title{
Association of Aortic Distensibility and Left Ventricular Function in Patients With Stenotic Bicuspid Aortic Valve and Preserved Ejection Fraction: A CMR study
}

\author{
Yijun Pan \\ Shanghai Medical University: Fudan University \\ Jiang Lin \\ Zhongshan Hospital Fudan University \\ Yongshi Wang \\ Zhongshan Hospital Fudan University \\ Jun Li \\ Zhongshan Hospital Fudan University \\ Pengju Xu \\ Zhongshan Hospital Fudan University \\ Mengsu Zeng \\ Zhongshan Hospital Fudan University \\ Yan Shan ( $\nabla$ shan.yan@zs-hospital.sh.cn ) \\ Zhongshan Hospital Fudan University https://orcid.org/0000-0003-0212-3242
}

\section{Research Article}

Keywords: Bicuspid aortic valve, Aortic stenosis, Aortic distensibility, left ventricular remodeling, myocardial strain, Cardiovascular magnetic resonance

Posted Date: December 15th, 2021

DOI: https://doi.org/10.21203/rs.3.rs-1165672/v1

License: (9) This work is licensed under a Creative Commons Attribution 4.0 International License. Read Full License

Version of Record: A version of this preprint was published at The International Journal of Cardiovascular Imaging on March 12th, 2022. See the published version at https://doi.org/10.1007/s10554-022-02581-4. 


\section{Abstract}

To determine the relationship between aortic distensibility and left ventricular (LV) remodeling, myocardial strain and blood biomarkers in patients with stenotic bicuspid aortic valve (BAV) and preserved ejection fraction (EF) by cardiovascular magnetic resonance (CMR). 43 stenotic BAV patients were prospectively selected for 3.0T CMR. Patients were divided into LV remodeling group (LV mass /volume $\geq 1.15, \mathrm{n}=21$ ) and non-remodeling group (LV mass/volume $<1.15, \mathrm{n}=22$ ). Clinical characteristics, biochemical data including cardiac troponin T(cTNT), N-terminal pro-B type natriuretic peptide (NT-proBNP) and creatine kinase isoenzyme were noted. Distensibility of middle ascending aorta (mid-AA) and proximal descending aorta, LV structural and functional parameters, global and regional myocardial strain were measured. Compared to non-remodeling group, LV remodeling group had significantly decreased LV global strain (radial: $26.04 \pm 8.70 \%$ vs. $32.92 \pm 7.81 \%, P=0.009$; circumferential: $-17.20 \pm 3.38 \%$ vs. $-19.65 \pm 2.34 \%, P=0.008$; longitudinal: $-9.13 \pm 2.34 \%$ vs. $-11.63 \pm 1.99 \%, P \llbracket 0.001$ ), while radial and circumferential strain were significantly reduced at the base (radial: $28.52 \pm 9.47 \%$ vs. $39.65 \pm 10.33 \%, P=0.001$; circumferential: $-14.45 \pm 2.97 \%$ vs. $-17.22 \pm 2.38 \%, P=0.002)$, longitudinal strain was significantly reduced at all regions (basal: $-5.79 \pm 3.43 \%$ vs. $-8.65 \pm 2.42 \%, P=0.003$; mid: $-8.62 \pm 2.21 \%$ vs. $-11.33 \pm 2.58 \%, P=0.001$; apical : $-12.79 \pm 2.49 \%$ vs. $-15.04 \pm 2.20 \%, P=0.003)$. In addition, mid-AA distensibility was independently associated with $L V$ remodeling $(\beta=-0.282, P=0.003)$, and it was also significantly correlated with LV global strain (radial: $r=0.392, P=0.009$; circumferential: $r=-0.348, P=0.022$; longitudinal: $r=-0.333, P=0.029)$, cTNT $(r=-0.333, P=0.029)$ and NT-proBNP $(r=-0.440, P=0.003)$. In this cohort with stenotic BAV and preserved EF, mid-AA distensibility is found significantly associated with LV dysfunction, which may be an important factor for predicting adverse cardiovascular events and a potential therapeutic target to prevent heart failure.

\section{Introduction}

Bicuspid aortic valve (BAV) is the most common congenital cardiovascular malformation in adult, affecting $0.5-2 \%$ of the population. Almost $50 \%$ of BAV subjects with severe aortic stenosis (AS) require surgery in their lifespan[1, 2]. BAV is characterized by a narrowed valve orifice and severe valve calcification, concomitant AS aggravates the degree of left ventricular (LV) outflow tract obstruction, resulting in increased LV afterload. Studies have indicated that stenotic BAV patients exhibited increased LV mass, depressed LV contractility and impaired diastolic function with marked hemodynamic alterations $[3,4]$. And notably, the impaired LV diastolic function is related to reduced aortic elasticity [57], supporting the hypothesis that BAV disease is not confined to valve but also affects aorta and left ventricle.

Aorta is recognized as a conduit for buffering blood pressure, and its abnormal elastic property is a major contributor to increased LV afterload. LV remodeling, the result of myocardial injury or overload, is manifested by alterations in geometry and structure, systolic and diastolic dysfunction and myocardial fibrosis[8]. LV remodeling has emerged as a key predictor of adverse cardiovascular events in BAV, especially for patients with significant valvular dysfunction $[9,10]$. However, there has been few reports so 
far documenting the relationship between aortic distensibility and LV remodeling and myocardial strain in patients with stenotic BAV and preserved ejection fraction (EF).

Cardiovascular magnetic resonance (CMR) is considered the gold standard for evaluating the structure and function of aorta and left ventricle owing to its superior temporal and spatial resolution and less operator dependence. Compared to simple measurements of LVEF, feature-tracking allows comprehensive and quantitative measurements of global and regional myocardial strain, which reflects early and subtle changes in subclinical LV dysfunction[11].

Therefore, the purpose of this study is to determine the relationship between aortic distensibility and LV remodeling, myocardial strain and blood biomarkers in stenotic BAV patients with preserved EF by using CMR.

\section{Methods}

\section{Study population}

BAV patients with moderated to severe AS and persevered EF were diagnosed by transthoracic echocardiography at our institution and prospectively recruited for CMR from September 2020 to November 2021. BAV patients were categorized into two groups according to LV remodeling index as follows: LV remodeling group (LV mass /volume $\geq 1.15, n=21$ ) and non-remodeling group (LV mass/volume $<1.15, \mathrm{n}=22$ ).

Moderated to severe AS was defined as peak velocity $\geq 3.0 \mathrm{~m} / \mathrm{s}$ and (or) mean transaortic pressure gradient $\geq 20 \mathrm{mmHg}$. Exclusion criteria were as follows: LVEF $₫ 50 \%$, other valvular dysfunction (including more than mild aortic insufficiency), aortic dissection, coronary artery disease and myocardial infarction, previous cardiac surgery. The present study conformed to the Declaration of Helsinki and was approved by the Ethics Committee of the Zhongshan Hospital Fudan University, Shanghai, China. Informed consent was obtained from all participants.

\section{Echocardiography}

All BAV patients underwent transthoracic echocardiography with a commercially available system (iE33, Philips Medical Systems, Bothell WA, USA). Standard 2D and Doppler echocardiographic examinations were performed at rest in the left lateral decubitus position according to ASE recommendations[12]. The diagnosis of BAV and aortic valve morphology was evaluated in the parasternal short-axis views with only two valve cusps clearly identified. Valve morphology was classified by leaflet fusion type, left and right cusp fusion type (LR), right and non-coronary cusp fusion type (RN) and left and non-coronary cusp fusion type (LN)[13]. Continuous-wave Doppler was used to measure the aortic valve peak velocity. Mean transaortic pressure gradient was calculated using the simplified Bernoulli equation.

\section{Aortic and CMR Imaging}


All examinations were performed on 3.0T scanner (Ingenia CX; Philips Healthcare, Best, The Netherlands) with 32-channel body phased-array surface coil. Aortic and LV images were acquired using ECG-gated steady state free precession acquisition with breath hold. The scanning parameters are as follows: TR 2.6-3.1 msec, TE 1.3-1.5 msec, flip angle $45^{\circ}$, slice thickness $8 \mathrm{~mm}, 2 \mathrm{~mm}$ section gap (for short-axis images), FOV $304 \mathrm{~mm} \times 304 \mathrm{~mm} \times 8 \mathrm{~mm}$, matrix $152 \times 225 \times 1$. The number of images was 25-30 per cardiac cycle. In cine short-axis view, 8-10 continuous images were obtained from mitral valve to LV apex. Cine images of two 2-chamber, 3-chamber and 4-chamber views were also acquired. Based on the transverse, coronal and sagittal positions, cine images of the middle ascending aorta (mid-AA) and proximal descending aorta (PDA) were obtained at the level of the pulmonary artery (Fig.1).

\section{Analysis}

LV structure and function analysis were performed with commercial postprocessing software (cvi42 v5.11, Circle Cardiovascular Imaging, Alberta, Canada). The short axis stack was analyzed automatically contouring the epicardial and endocardial borders from base to apex at end-diastole and end-systole with manually correction when necessary (Fig.2). LVEF, LV end-systolic volume (LVESV), LV end- diastolic volume (LVEDV), LV stroke volume (LVSV), LV mass (LVM) were quantified and indexed to the body surface area (LVESVi, LVEDVi, LVSVi and LVMi, respectively) using the Mosteller formula. The trabeculae and papillary muscles were included. LV remodeling index was defined as LVM/LVEDV.

MR cine images (short-axis, 2-chamber, 3-chamber and 4-chamber views) were input to the Tissue Tracking module for myocardial strain analysis according to the $16 \mathrm{AHA}$ segmentation model. The borders of LV endocardium and epicardium were automatically delineated with manually correction when necessary. Global and regional (basal, mid, apical) strain values in radial, circumferential and longitudinal directions were obtained.

\section{Aortic Distensibility Analysis}

Maximum and minimum aortic cross-sectional areas and diameter over the cardiac cycle were determined on the postprocessing software (IntelliSpacePortal version 9.0.4; Philips Healthcare, Best, The Netherlands). Aortic distensibility was calculated by the following equation[14]: (Amax-Amin)/ Amin $\times$ (systolic-diastolic blood pressure). Amax and Amin are the maximum systolic aortic area and the minimum diastolic area, respectively. The brachial artery systolic and diastolic blood pressure were measured before CMR examination.

\section{Laboratory testing}

Venous blood samples were obtained before CMR examinations for all BAV patients. Cardiac troponin T(cTNT), N-terminal pro-B type natriuretic peptide (NT-proBNP), creatine kinase isoenzyme (CK-MB) levels were measured using the Elecsys Electro-chemo luminescent assay (Cobase 411 analyzer, Roche Diagnostics, Mannheim, Germany) in our laboratory department.

\section{Statistical analysis}


Analysis was performed with SPSS 20.0 software (Chicago IL, USA). Normality of data distribution was determined using the histogram and Shapiro-Wilk test. Data were reported as mean \pm SD or medians (interquartile ranges) for continuous variables and as frequencies (percentages) for categorical variables. Differences between two groups were assessed by the Student's T test (Gaussian distribution) or MannWhitney U-test (non-Gaussian distribution). Differences in percentages were evaluated using the chisquare tests or Fisher's exact tests. Relationship between aortic distensibility and LV global strain, blood biomarkers were assessed by Pearson's correlation coefficient. Data on cTNT and NT-proBNP were skewed and thus logarithmically transformed. Statistical significance was defined as 2 -sided $p<0.05$. Multivariate linear regression analysis was performed to determine the independent predictor of LV remodeling. Variables were entered in the model if $p<0.10$ in univariate analyses. To avoid collinearity between peak velocity and mean transaortic pressure gradient, only peak velocity was entered in the multivariate models. Intra- and interobserver reliabilities were evaluated by the intraclass correlation (ICC).

\section{Results}

\section{Baseline characteristics}

The demographics and biochemical data of all subjects were summarized in Table 1. There were no differences in age, gender, body mass index, systolic blood pressure, diastolic blood pressure, pulse pressure, heart rate, comorbid condition, medication status and valve phenotype in BAV patients. Compared to non-remodeling group, LV remodeling group had greater mean transvalvular pressure gradient $(60.24 \pm 19.69 \mathrm{~mm} \mathrm{Hg}$ vs. $45.36 \pm 12.01 \mathrm{~mm} \mathrm{Hg}, P=0.005)$, higher peak velocity $(4.86 \pm 0.64 \mathrm{~m} / \mathrm{s}$ vs. $4.37 \pm 0.52 \mathrm{~m} / \mathrm{s}, \mathrm{P}=0.009)$ and elevated NT-proBNP level (593.80 (238.85-792.25) pg/ml vs. 110.20 (78.15204.33) $\mathrm{pg} / \mathrm{ml}, \mathrm{P}<0.001)$. There was no significant difference in cTNT and CK-MB between two groups. 
Table 1

Baseline Characteristics and biochemical data in BAV patients

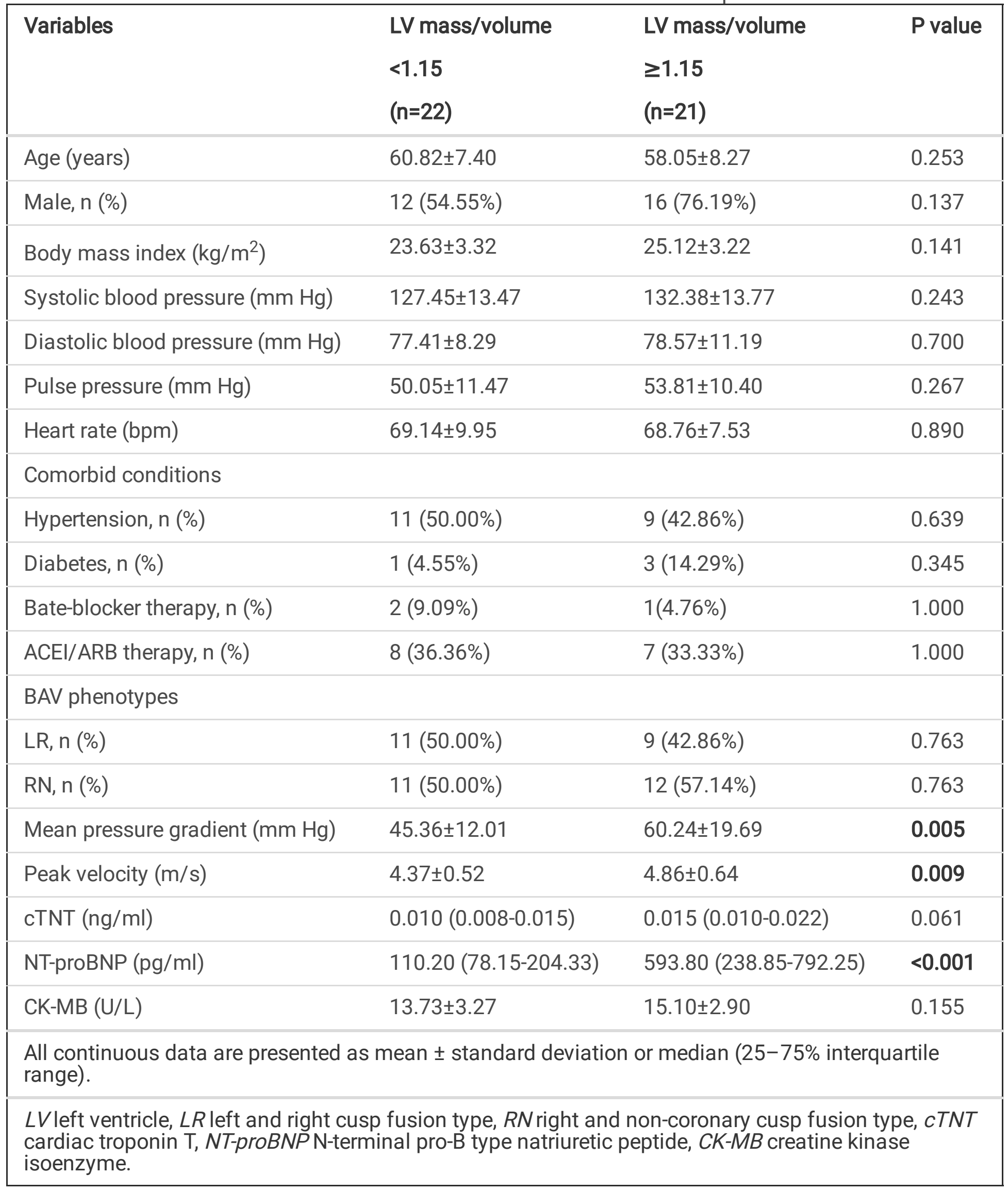

\section{LV parameters}


There were no differences in LVEF, LVEDVi, LVESVi and LVSVi in BAV patients. The LV remodeling group demonstrated significantly higher LVMi $(97.50 \pm 23.45 \mathrm{~g} / \mathrm{m} 2$ vs. $66.61 \pm 20.08 \mathrm{~g} / \mathrm{m} 2, P<0.001)$ and LV mass/volume $(1.29 \pm 0.21 \mathrm{~g} / \mathrm{ml}$ vs. $0.91 \pm 0.12 \mathrm{~g} / \mathrm{ml}, \mathrm{P}<0.001)$ than non-remodeling group (Table 2$)$.

BAV patients with LV remodeling had significantly lower global radial (GRS) $(26.04 \pm 8.70 \%$ vs. $32.92 \pm 7.81 \%, P=0.009)$, global circumferential (GCS) $(-17.20 \pm 3.38 \%$ vs. $-19.65 \pm 2.34 \%, P=0.008)$ and global longitudinal strain (GLS) $(-9.13 \pm 2.34 \%$ vs. $-11.63 \pm 1.99 \%, P<0.001)$ than those without LV remodeling. Moreover, the distribution of regional myocardial impairment is heterogeneously from base to apex, marked in radial and circumferential at base (radial: $28.52 \pm 9.47 \%$ vs. $39.65 \pm 10.33 \%, P=0.001$; circumferential: $-14.45 \pm 2.97 \%$ vs. $-17.22 \pm 2.38 \%, P=0.002$ ), slightly at middle (radial: $25.33 \pm 10.26 \%$ vs. $31.29 \pm 7.53 \%, P=0.035$; circumferential: $-18.03 \pm 4.07 \%$ vs. $-20.22 \pm 2.79 \%, P=0.045$ ), and no significant difference at apex (Table 2). For longitudinal strain, significant differences were seen from base to apex (base: $-5.79 \pm 3.43 \%$ vs. $-8.65 \pm 2.42 \%, P=0.003$; middle: $-8.62 \pm 2.21 \%$ vs. $-11.33 \pm 2.58 \%, P=0.001$; apex: $-12.79 \pm 2.49 \%$ vs. $-15.04 \pm 2.20 \%, P=0.003$ ). 
Table 2

Left Ventricular Parameters in BAV patients

\begin{tabular}{|c|c|c|c|}
\hline Variables & $\begin{array}{l}\text { LV mass/volume } \\
<1.15 \\
(n=22)\end{array}$ & $\begin{array}{l}\text { LV mass/volume } \\
\geq 1.15 \\
(n=21)\end{array}$ & $P$ value \\
\hline \multicolumn{4}{|c|}{ LV volumes, mass and function } \\
\hline LVEF (\%) & $68.73 \pm 5.80$ & $67.88 \pm 6.27$ & 0.647 \\
\hline LVEDVi $\left(\mathrm{ml} / \mathrm{m}^{2}\right)$ & $75.33 \pm 13.45$ & $76.18 \pm 14.23$ & 0.840 \\
\hline LVESVi $\left(\mathrm{ml} / \mathrm{m}^{2}\right)$ & $23.79 \pm 6.45$ & $24.15 \pm 5.51$ & 0.842 \\
\hline LVSVi $\left(\mathrm{ml} / \mathrm{m}^{2}\right)$ & $52.19 \pm 9.91$ & $51.65 \pm 11.86$ & 0.871 \\
\hline LVMi $\left(g / m^{2}\right)$ & $66.61 \pm 20.08$ & $97.50 \pm 23.45$ & $<0.001$ \\
\hline LV mass/volume (g/ml) & $0.91 \pm 0.12$ & $1.29 \pm 0.21$ & $<0.001$ \\
\hline \multicolumn{4}{|l|}{ LV strain } \\
\hline \multicolumn{4}{|l|}{ Radial strain (\%) } \\
\hline - basal & $39.65 \pm 10.33$ & $28.52 \pm 9.47$ & 0.001 \\
\hline - mid & $31.29 \pm 7.53$ & $25.33 \pm 10.26$ & 0.035 \\
\hline - apical & $31.41 \pm 10.41$ & $28.07 \pm 11.05$ & 0.314 \\
\hline - global & $32.92 \pm 7.81$ & $26.04 \pm 8.70$ & 0.009 \\
\hline \multicolumn{4}{|l|}{ Circumferential strain (\%) } \\
\hline - basal & $-17.22 \pm 2.38$ & $-14.45 \pm 2.97$ & 0.002 \\
\hline - mid & $-20.22 \pm 2.79$ & $-18.03 \pm 4.07$ & 0.045 \\
\hline - apical & $-21.86 \pm 3.74$ & $-19.39 \pm 4.50$ & 0.056 \\
\hline - global & $-19.65 \pm 2.34$ & $-17.20 \pm 3.38$ & 0.008 \\
\hline \multicolumn{4}{|l|}{ Longitudinal strain (\%) } \\
\hline - basal & $-8.65 \pm 2.42$ & $-5.79 \pm 3.43$ & 0.003 \\
\hline- mid & $-11.33 \pm 2.58$ & $-8.62 \pm 2.21$ & 0.001 \\
\hline
\end{tabular}

All continuous data are presented as mean \pm standard deviation.

LVEF left ventricular ejection fraction, $L V E D V i$ left ventricular end-diastolic volume index, $L V E S V i$ left ventricular end-systolic volume index, LVSVi left ventricular stroke volume index, LVMi left ventricular mass index. 


\begin{tabular}{|llll|}
\hline Variables & LV mass/volume & LV mass/volume & P value \\
& $<1.15$ & $\geq 1.15$ & \\
& $(\mathbf{n = 2 2})$ & $(\mathbf{n = 2 1 )}$ & \\
\hline - apical & $-15.04 \pm 2.20$ & $-12.79 \pm 2.49$ & $\mathbf{0 . 0 0 3}$ \\
\hline - global & $-11.63 \pm 1.99$ & $-9.13 \pm 2.34$ & $<0.001$ \\
\hline All continuous data are presented as mean \pm standard deviation. & & \\
\hline $\begin{array}{l}\text { LVEF left ventricular ejection fraction, } \\
\text { ventricular end-systolic volume index, } L V S V i \text { left ventricular stroke volume index, } \\
\text { mass index. }\end{array}$ & & \\
\hline
\end{tabular}

\section{Aortic Distensibility}

Though mid-AA diameter was similar between two groups, distensibility of mid-AA was lower in LV remodeling group than non-remodeling group $\left(1.22 \pm 0.2410^{-3} \mathrm{~mm} \mathrm{Hg}^{-1}\right.$ vs $1.60 \pm 0.4110^{-3} \mathrm{~mm} \mathrm{Hg}^{-1}$, $\mathrm{P}=0.001$ ). There were no differences in diameter and distensibility of PDA among two groups (Table 3 ).

Table 3

Aortic Diameter and Distensibility in BAV patients

\begin{tabular}{|llll|}
\hline Variables & $\begin{array}{l}\text { LV mass/volume } \\
<1.15\end{array}$ & $\begin{array}{l}\text { LV mass/volume } \\
(\mathbf{n = 2 2})\end{array}$ & P value \\
& $\begin{array}{l}\mathbf{( n = 2 1 )} \\
\end{array}$ & $4.09 \pm 0.52$ & \\
\hline Mid-AA diameter $(\mathrm{cm})$ & $1.60 \pm 0.41$ & $1.22 \pm 0.24$ & 0.382 \\
\hline Mid-AA distensibility $\left(10^{-3} \mathrm{~mm} \mathrm{Hg}^{-1}\right)$ & $2.38 \pm 0.25$ & $2.45 \pm 0.17$ & 0.302 \\
\hline PDA diameter $(\mathrm{cm})$ & $2.42 \pm 0.76$ & $2.22 \pm 0.66$ & 0.339 \\
\hline PDA distensibility $\left(10^{-3} \mathrm{~mm} \mathrm{Hg}^{-1}\right)$ & & \\
\hline All continuous data are presented as mean \pm standard deviation. & \\
\hline Mid-AA middle ascending aorta, $P D A$ proximal descending aorta. & \\
\hline
\end{tabular}

\section{Univariable and Multivariable Predictors of LV Remodeling}

Univariate and multivariate analysis results are shown in Table 4, gender, body mass index, pulse pressure, mean transvalvular pressure gradient, peak velocity and mid-AA distensibility were correlated with LV remodeling, presented as LV mass/volume. On multivariable regression, only body mass index ( $\beta$ $=0.021, P=0.020)$, peak velocity $(\beta=0.112, P=0.024)$ and mid-AA distensibility $(\beta=-0.282, P=0.003)$ were independently associated with LV remodeling $\left(R^{2}=0.769\right)$.

Table 4 Univariate and multivariate predictors of LV remodeling in BAV Patients 


\begin{tabular}{lccccc}
\hline Variables & \multicolumn{2}{c}{ Unadjusted Correlates of } & \multicolumn{2}{c}{ Multivariate Correlates of } \\
& \multicolumn{2}{c}{ LV remodeling } & \multicolumn{3}{c}{ LV remodeling } \\
& $\rho$ & P value & $\beta$ & $95 \%(\mathrm{CI})$ & P value \\
\hline Man & -0.185 & $\mathbf{0 . 0 2 3}$ & -0.134 & $-0.272,0.004$ & 0.057 \\
Age & -0.005 & 0.375 & & & \\
Body mass index & 0.026 & $\mathbf{0 . 0 3 1}$ & 0.021 & $0.004,0.039$ & $\mathbf{0 . 0 2 0}$ \\
Systolic blood pressure & 0.003 & 0.282 & & & \\
Diastolic blood pressure & -0.003 & 0.437 & & & \\
Pulse pressure & 0.007 & $\mathbf{0 . 0 3 9}$ & 0.002 & $-0.004,0.008$ & 0.516 \\
Heart rate & 0.003 & 0.493 & & & \\
Mean pressure gradient & 0.007 & $\mathbf{0 . 0 0 2}$ & & & \\
Peak velocity & 0.192 & $\mathbf{0 . 0 0 2}$ & 0.112 & $0.016,0.209$ & $\mathbf{0 . 0 2 4}$ \\
Mid-AA distensibility & -0.409 & $\mathbf{6 . 0 0 1}$ & -0.282 & $-0.459,-0.106$ & $\mathbf{0 . 0 0 3}$ \\
PDA distensibility & -0.078 & 0.165 & & & \\
\hline
\end{tabular}

$R^{2}=0.769$

\section{Correlation of Aortic Distensibility with Other Variables}

GRS was positively and significantly correlated with mid-AA distensibility ( $r=0.392, P=0.009$, Fig. 4A), while GCS $(r=-0.348, P=0.022$, Fig. 4B) and GLS $(r=-0.333, P=0.029$, Fig. $4 C)$ were negatively correlated with mid-AA distensibility. Log CTNT ( $r=-0.333, P=0.029$, Fig. 4D) and Log NT-proBNP $(r=-0.440, P=0.003$, Fig. 4E) were negatively correlated with mid-AA distensibility. CK-MB was not significantly correlated with mid-AA distensibility. There was no significant correlation between PDA distensibility and Log cTNT, Log NT-proBNP, CK-MB, GRS, GCS, GLS.

\section{Reproducibility}

Intra- and interobserver variabilities of MRI parameters (aortic distensibility, LVEDVi, LVMi, GRS, GCS, GLS) were obtained with 26 random cases by two observers. The intra- and inter-observer variability results were shown in Table 5. All the MRI parameters demonstrated good agreements. 
Table 5

Inter- and Intraobserver Analyses for MRI parameters

\begin{tabular}{|lllll|}
\hline & \multicolumn{2}{l}{ Intraobserver } & \multicolumn{2}{l|}{ Interobserver } \\
\hline & ICC & $95 \% \mathrm{Cl}$ & ICC & $95 \% \mathrm{Cl}$ \\
\hline Mid-AA distensibility & 0.921 & $0.834,0.964$ & 0.959 & $0.910,0.982$ \\
\hline PDA distensibility & 0.925 & $0.842,0.966$ & 0.961 & $0.914,0.983$ \\
\hline LVEDVi & 0.968 & $0.747,0.990$ & 0.984 & $0.855,0.995$ \\
\hline LVMi & 0.977 & $0.947,0.990$ & 0.988 & $0.973,0.990$ \\
\hline GRS & 0.926 & $0.763,0.971$ & 0.961 & $0.865,0.986$ \\
\hline GCS & 0.935 & $0.783,0.975$ & 0.966 & $0.878,0.987$ \\
\hline GLS & 0.901 & $0.757,0.958$ & 0.948 & $0.862,0.978$ \\
\hline GRS: global radial strain; GCS: global circumferential strain; GLS: global longitudinal strain. \\
\hline
\end{tabular}

\section{Discussion}

Current ESC/EACTS guideline has formulated strong recommendations for early intervention in patients with symptomatic severe AS or those who are asymptomatic but with impaired LV function (LVEF $<50 \%$ ) [15]. Management of asymptomatic patients with moderate to severe AS and preserved EF is otherwise controversial $[16,17]$. BAV with moderate to severe AS is more prevalent in young individuals and is associated with ascending aorta dilatation, aneurysm formation and dissection. Moreover, BAV tends to have a higher degree of LV outflow tract obstruction than the tricuspid aortic valve (TAV) with AS, making it difficult to determine the appropriate interventions and their timing. The present study focused exclusively on stenotic BAV patients with preserved EF and for the first time, identified the significant association between mid-AA distensibility and LV dysfunction by means of CMR, providing an additional method for assessing early and subtle ventricular dysfunction.

In this study, we utilized CMR strain-based technique to characterize the global and regional strain. We found a reduction of global myocardial strain in BAV patients with LV remolding, which is consistent with prior echocardiography studies $[4,9]$. We further demonstrated that the difference in strain was prominent in the base of the heart, which has large amount of myocardial fibers of the LV. This pattern of systolic dysfunction is similar to that seen in dilated cardiomyopathy[18] and isolated AS[19]. The asymmetric distribution of myocardial fibrosis may contribute to the heterogeneous contractile injury in patients with LV outflow tract obstruction. It should be noted that, LV remodeling group exhibited significantly decreased strain from base to apex longitudinally when compared to non-remodeling group. GLS has emerged as a more sensitive and objective marker to quantify subendocardial contraction. William et al. [9] reported that impaired GLS was independently associated with adverse cardiovascular events during a median follow-up period of 36 months in BAV patients. In 294 patients with severe AS, Ng et al.[20] 
revealed that impaired GLS was an independent predictor of all-cause mortality. Thus, tissue tracking technique may facilitate early detection of abnormal LV strain in sentinel regions and identify stenotic BAV patients who might benefit from earlier surgical interventions.

Elastic fiber degeneration and extracellular matrix disorder are the major pathogenic mechanism for the impairment of aortic elasticity in stenotic BAV patients [21,22]. In the results of our study, ascending aorta was involved earlier and more obviously, largely due to its high elastin to collagen ratio[23]. It was particularly noticeable that mid-AA distensibility was a significant predictor of $L V$ remodeling in patients with stenotic BAV and preserved EF. This association was independent of blood pressure and is similar to the findings reported by Rider et al. in hypertension patients[24]. It is possible that with the decreased aortic elasticity, accelerated reflected pressure wave arrives in late systole, leading to increased LV afterload, subendocardial ischemia, increased oxygen demand and further interstitial fibrosis[25-27]. The present study supports the hypothesis that aortic stiffening increases the risk of poorer cardiovascular events in stenotic BAV patients through adverse LV remodeling. BAV with AS should be diagnosed at an early stage when LV and aortic remodeling are more likely to be reversible. Optimal medical therapy, especially angiotensin-converting enzyme inhibitor and angiotensin receptor blocker, may have favorable effects before the onset of LV dysfunction by modifying aortic stiffness.

This study also demonstrated that increased aortic stiffening was associated with reduced global myocardial strain in stenotic BAV patients with preserved EF, which is similar to the echocardiography findings in patients with chronic kidney disease[28] and type 2 diabetes[29]. Additionally, the upregulation of cTNT and NT-proBNP after myocardial dysfunction is closely linked to activation of inflammatory cytokines, oxidative stress and myocardial wall stretch[30,31]. The present study showed a significant relationship between mid-AA distensibility and CTNT, NT-proBNP, whose prognostic value has been proven in AS patients[32, 33], indicating that stenotic BAV patients with reduced aortic elasticity were probably at a more advanced disease stage.

\section{Study Limitations}

This was a cross-sectional study with a small sample size, and the cause-effect relationship between aortic and LV remodeling cannot be identified. Therefore, further longitudinal researches with larger sample sizes may be needed. In addition, we did not recruit stenotic TAV as controls, since these patients are older and complicated with more cardiovascular risk factors ${ }^{[34]}$, which may cause bias into the study.

\section{Conclusions}

The detection of abnormal strain in longitudinal or radial and circumferential at base may aid in identifying sub-clinical LV dysfunction. In line with the concept of ventricular vascular coupling, this CMR study further confirms that mid-AA distensibility is independently associated with LV remodeling. Mid-AA distensibility may therefore represent a valuable indicator for optimized risk stratification, appropriate timing of intervention for stenotic BAV patients with preserved EF.

Page $12 / 19$ 


\section{Declarations}

Authors' contributions Yijun Pan-data collection, analysis, manuscript draft; Jiang Lin -study conception and design, manuscript review; Yongshi Wang, Jun Li -data collection, analysis; Pengju Xu, Mengsu Zeng -manuscript review; Yan Shan-study conception and design, data collection, manuscript review. All authors read and approved the final manuscript.

Funding This work was supported by National Natural Science Foundation of China (Grant Number:81901818 and 82071991) and Science and Technology Commission of Shanghai Municipality, China (Grant Number:19ZR1451000).

\section{Compliance with ethical standards}

Conflict of interest All authors declare that they have no conflict of interest.

Ethical approval This study was performed in line with the principles of the Declaration of Helsinki. Approval was granted by the local ethics committee of our institution (NO: B2020-232).

Informed consent Informed consent was obtained from all individual participants included in the study.

\section{References}

1. Michelena HI, Prakash SK, Della Corte A et al (2014) Bicuspid Aortic Valve Identifying Knowledge Gaps and Rising to the Challenge From the International Bicuspid Aortic Valve Consortium (BAVCon). Circulation 129(25):2691-2704

2. Hoffman JIE, Kaplan S (2002) The incidence of congenital heart disease. J Am Coll Cardiol 39(12):1890-1900

3. Stefek H, Berhane H, Robinson J et al (2019) Comprehensive MR Analysis of Cardiac Function, Aortic Hemodynamics and Left Ventricular Strain in Pediatric Cohort with Isolated Bicuspid Aortic Valve. Pediatr Cardiol 40(7):1450-1459

4. Zhang X, Zhu M, He T et al (2015) Cardiac Mechanics in Isolated Bicuspid Aortic Valve Disease With Normal Ejection Fraction: A Study of Various Valvular Lesion Types. Medicine (Baltimore), 94(47): e2085

5. Nucifora G, Miller J, Gillebert C et al (2018) Ascending Aorta and Myocardial Mechanics in Patients with "Clinically Normal" Bicuspid Aortic Valve Insights from Cardiovascular Magnetic Resonance Tissue-Tracking Imaging. Int Heart J 59(4):741-749

6. Santarpia G, Scognamiglio G, Di Salvo G et al (2012) Aortic and left ventricular remodeling in patients with bicuspid aortic valve without significant valvular dysfunction: a prospective study. Int $J$ Cardiol 158(3):347-352

7. Lee SY, Shim CY, Hong G-R et al (2015) Association of Aortic Phenotypes and Mechanical Function With Left Ventricular Diastolic Function in Subjects With Normally Functioning Bicuspid Aortic 
Valves and Comparison to Subjects With Tricuspid Aortic Valves. Am J Cardiol 116(10):1547-1554

8. Von Knobelsdorff-Brenkenhoff F, Karunaharamoorthy A, Trauzeddel RF et al (2016) Evaluation of Aortic Blood Flow and Wall Shear Stress in Aortic Stenosis and Its Association With Left Ventricular Remodeling. Circ -Cardiovasc Imaging 9(3):e004038

9. Kong WKF, Vollema EM, Prevedello F et al (2020) Prognostic implications of left ventricular global longitudinal strain in patients with bicuspid aortic valve disease and preserved left ventricular ejection fraction. Eur Heart J -Cardiovasc Imaging 21(7):759-767

10. Geiger J, Rahsepar AA, Suwa K et al (2018) 4D flow MRI, cardiac function, and T1-mapping: Association of valve-mediated changes in aortic hemodynamics with left ventricular remodeling. $J$ Magn Reson Imaging 48(1):121-131

11. Claus P, Omar A, Pedrizzetti G et al (2015) Tissue Tracking Technology for Assessing Cardiac Mechanics: Principles, Normal Values, and Clinical Applications [J]. JACC-Cardiovasc Imag 8(12):1444-1460

12. Lang (2016) Recommendations for Cardiac Chamber Quantification by Echocardiography in Adults: An Update from the American Society of Echocardiography and the European Association of, Cardiovascular Imaging (vol 16, pg 233, 2015). Eur Heart J -Cardiovasc Imaging 17(4):412-412

13. Sievers $\mathrm{H}-\mathrm{H}$, Schmidtke $\mathrm{C}$ (2007) A classification system for the bicuspid aortic valve from 304 surgical specimens. J Thorac Cardiovasc Surg 133(5):1226-1233

14. O'rourke MF, Staessen JA, Vlachopoulos C et al (2002) Clinical applications of arterial stiffness; definitions and reference values. Am J Hypertens 15(5):426-444

15. Vahanian A, Beyersdorf F, Praz F et al (2021) 2021 ESC/EACTS Guidelines for the management of valvular heart disease. Eur Heart J DIO. 10.1093/eurheartj/ehab395

16. Chiam PTL, Ewe SH (2016) The expanding indications of transcatheter aortic valve implantation. Futur Cardiol 12(2):209-219

17. Makkar RR, Yoon S-H, Chakravarty T et al (2021) Association Between Transcatheter Aortic Valve Replacement for Bicuspid vs Tricuspid Aortic Stenosis and Mortality or Stroke Among Patients at Low Surgical Risk. JAMA-J Am Med Assoc 326(11):1034-1044

18. Joseph S, Moazami N, Cupps BP et al (2009) Magnetic Resonance Imaging-based Multiparametric Systolic Strain Analysis and Regional Contractile Heterogeneity in Patients With Dilated Cardiomyopathy. J Heart Lung Transplant 28(4):388-394

19. Weidemann F, Herrmann S, Stoerk S et al (2009) Impact of Myocardial Fibrosis in Patients With Symptomatic Severe Aortic Stenosis. Circulation 120(7):577-584

20. Ng ACT, Prihadi EA, Antoni ML et al (2018) Left ventricular global longitudinal strain is predictive of all-causemortality independent of aortic stenosis severity and ejection fraction. Eur Heart $\mathrm{J}$ Cardiovasc Imaging 19(8):859-867

21. Blunder S, Messner B, Aschacher T et al (2012) Characteristics of TAV-and BAV-associated thoracic aortic aneurysms-Smooth muscle cell biology, expression profiling, and histological analyses. Atherosclerosis 220(2):355-361 
22. Bollache E, Guzzardi DG, Sattari S et al (2018) Aortic valve-mediated wall shear stress is heterogeneous and predicts regional aortic elastic fiber thinning in bicuspid aortic valve-associated aortopathy. J Thorac Cardiovasc Surg 156(6):2112-2120e2112

23. Halloran B, Davis V, Mcmanus B et al (1995) Localization of aortic disease is associated with intrinsic differences in aortic structure. J Surg Res 59(1):17-22

24. Rider O, Nethononda R, Petersen S et al (2013) Concentric left ventricular remodeling and aortic stiffness: a comparison of obesity and hypertension. Int J Cardiol 167(6):2989-2994

25. Roman M, Okin P, Kizer J et al (2010) Relations of central and brachial blood pressure to left ventricular hypertrophy and geometry: the Strong Heart Study. J Hypertens 28(2):384-388

26. Chirinos J, Segers P, Hughes T et al (2019) Large-Artery Stiffness in Health and Disease: JACC Stateof-the-Art Review. J Am Coll Cardiol 74(9):1237-1263

27. Weber T, Chirinos J (2018) Pulsatile arterial haemodynamics in heart failure. Eur Heart J 39(43):3847-3854

28. Krishnasamy R, Hawley CM, Stanton T et al (2015) Left ventricular global longitudinal strain is associated with cardiovascular risk factors and arterial stiffness in chronic kidney disease.BMC Nephrol16(106)

29. Mehta S, Khoury PR, Madsen NL et al (2018) Arterial Thickness and Stiffness Are Independently Associated with Left Ventricular Strain. J Am Soc Echocardiogr 31(1):99-104

30. Kociol RD, Pang PS, Gheorghiade M et al (2010) Troponin Elevation in Heart Failure Prevalence, Mechanisms, and Clinical Implications. J Am Coll Cardiol 56(14):1071-1078

31. Vanderheyden M, Goethals $M$, Verstreken $S$ et al (2004) Wall stress modulates brain natriuretic peptide production in pressure overload cardiomyopathy. J Am Coll Cardiol 44(12):2349-2354

32. Zhang B, Xu H, Zhang H et al (2020) Prognostic Value of N-Terminal Pro-B-Type Natriuretic Peptide in Elderly Patients With Valvular Heart Disease. J Am Coll Cardiol 75(14):1659-1672

33. Lim P, Monin J, Monchi M et al (2004) Predictors of outcome in patients with severe aortic stenosis and normal left ventricular function: role of B-type natriuretic peptide. Eur Heart J 25(22):2048-2053

34. Sia CH, Ho JS, Chua JJ et al (2020) Comparison of Clinical and Echocardiographic Features of Asymptomatic Patients With Stenotic Bicuspid Versus Tricuspid Aortic Valves. Am J Cardiol 128:210-215

\section{Figures}



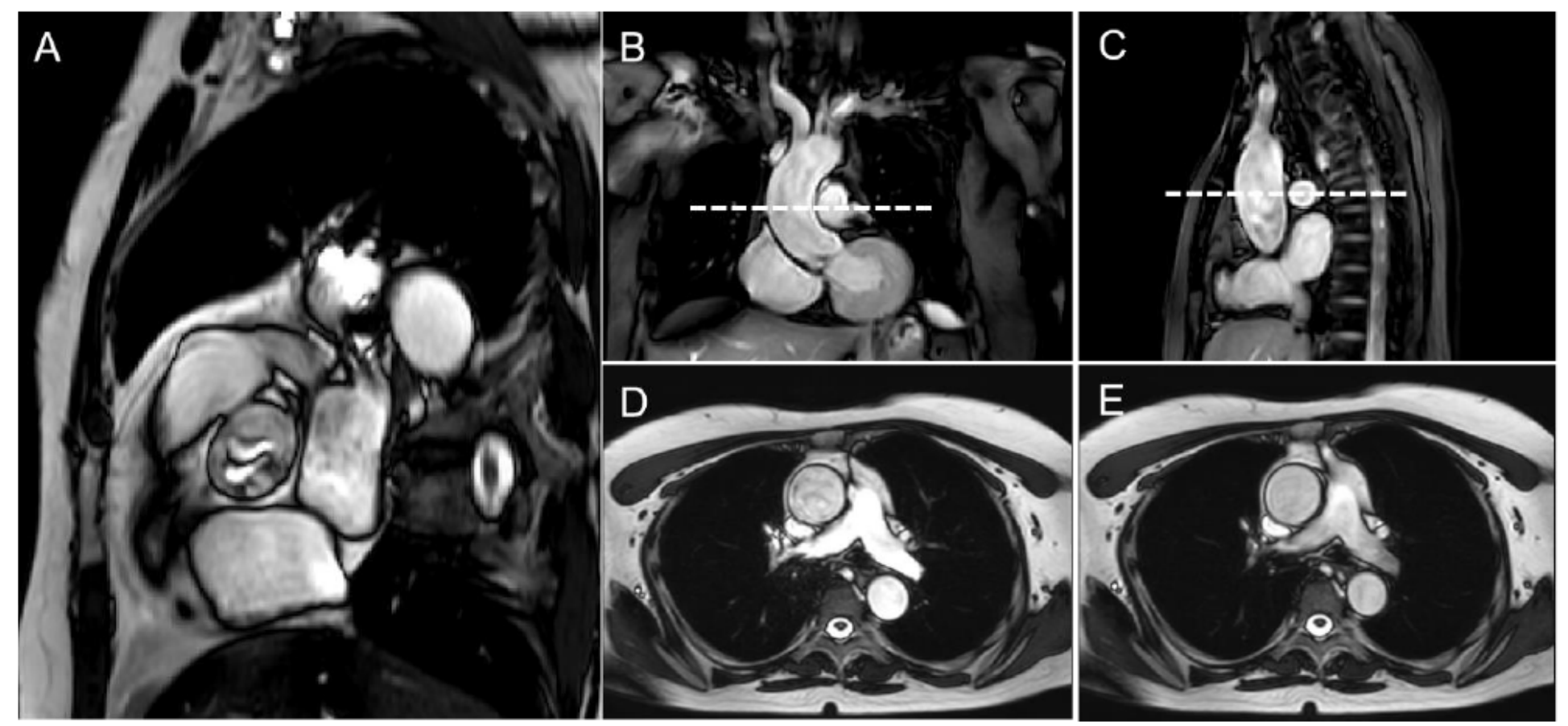

Figure 1

Examples of measuring distensibility of mid-AA and PDA. An BAV patient with moderate to severe AS and RN fusion (Plane A). Coronal (Plane B) and sagittal planes (Plane $C$ ) of the aorta are used to select the level of right pulmonary artery (dashed line) and measure diameter and cross-sectional area of mid-AA and PDA; Images of mid-AA and PDA acquired during systole (Plane D) and diastole (Plane E). RN: right and non-coronary cusp fusion type; Mid-AA: middle ascending aorta; PDA: proximal descending aorta. 
A
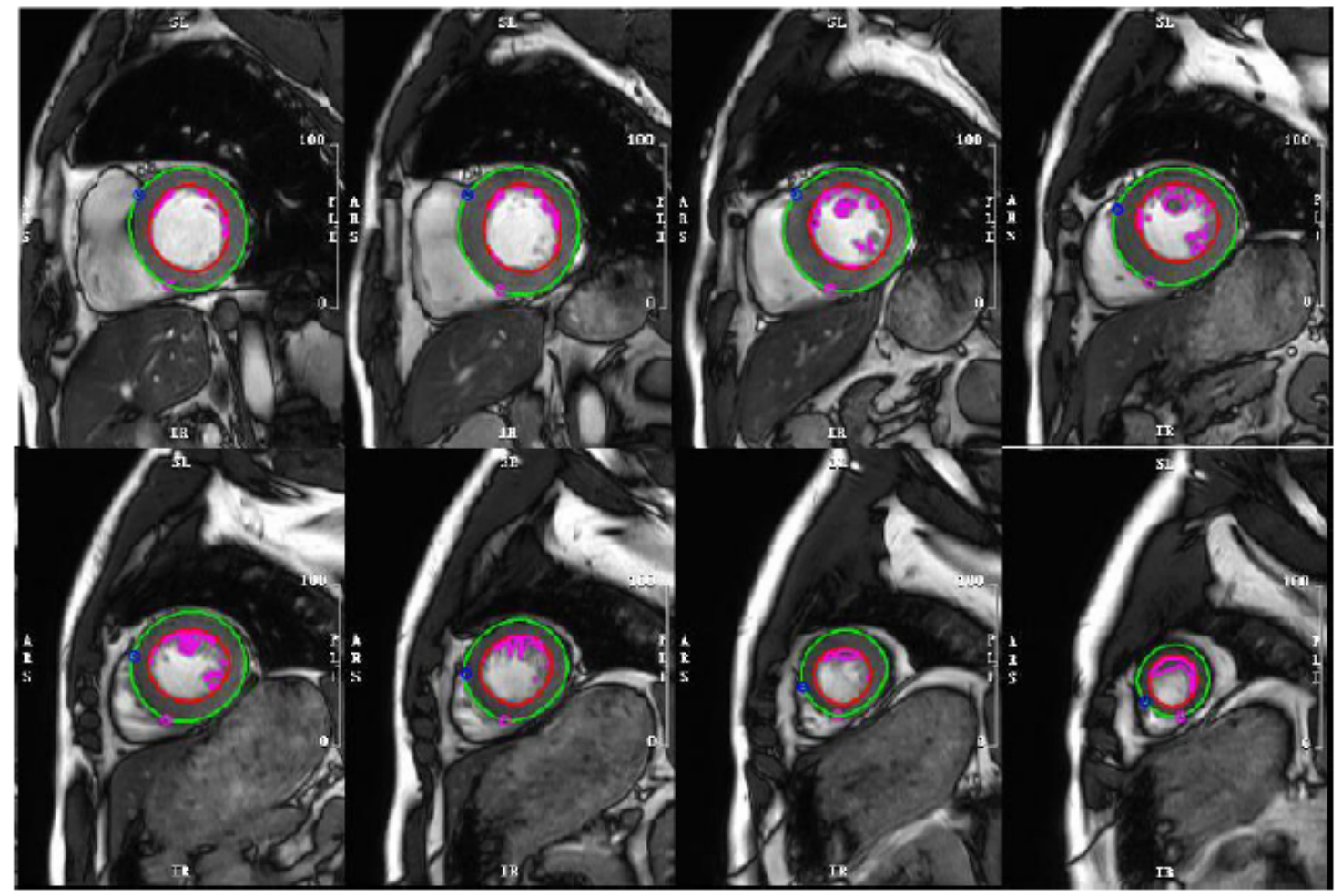

B

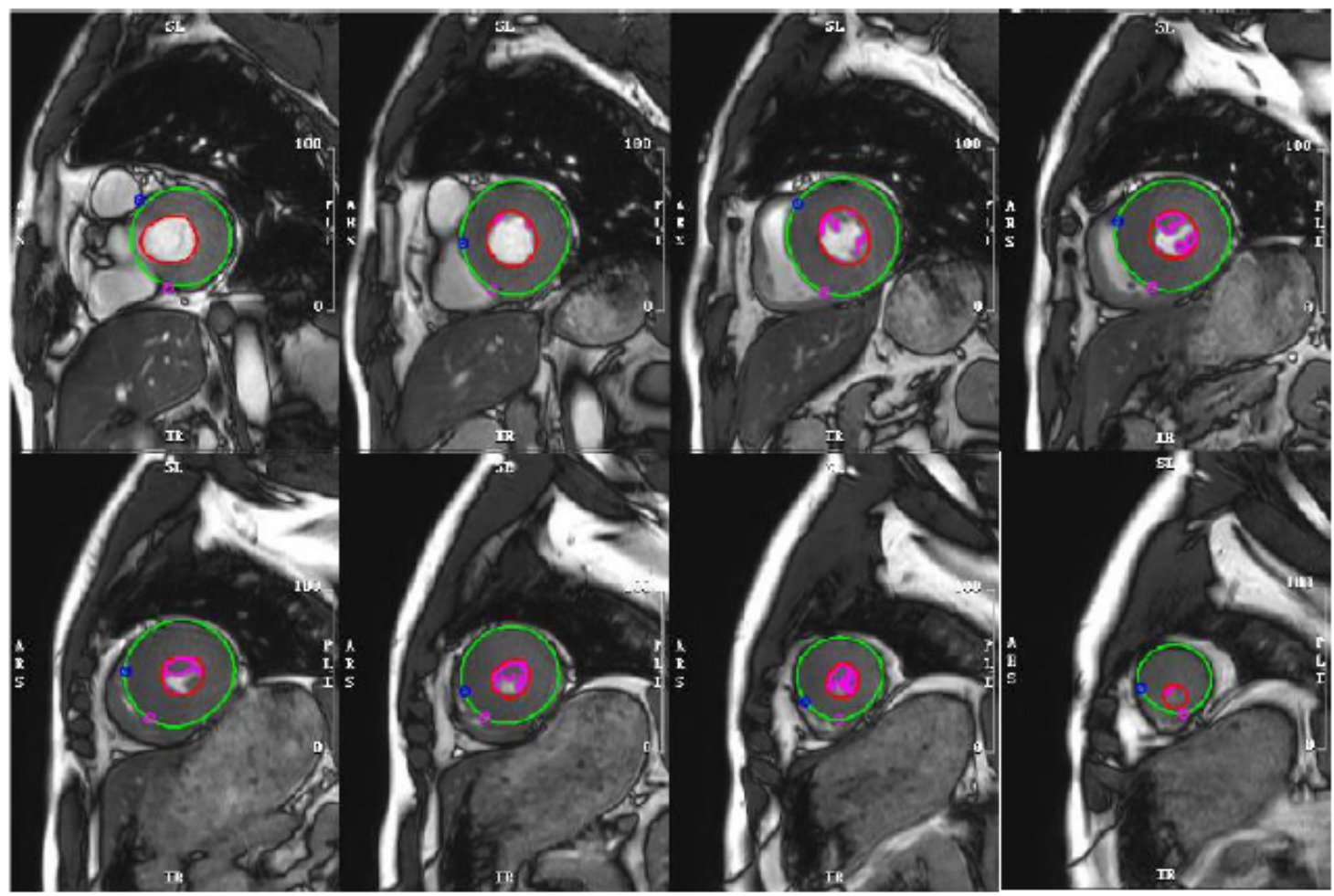

Figure 2

Examples of LV structure and function analysis on the short-axis cine images. The trace of LV endocardial and epicardial contours from base to apex at end-diastole (Plane A) and end-systole (Plane B). 

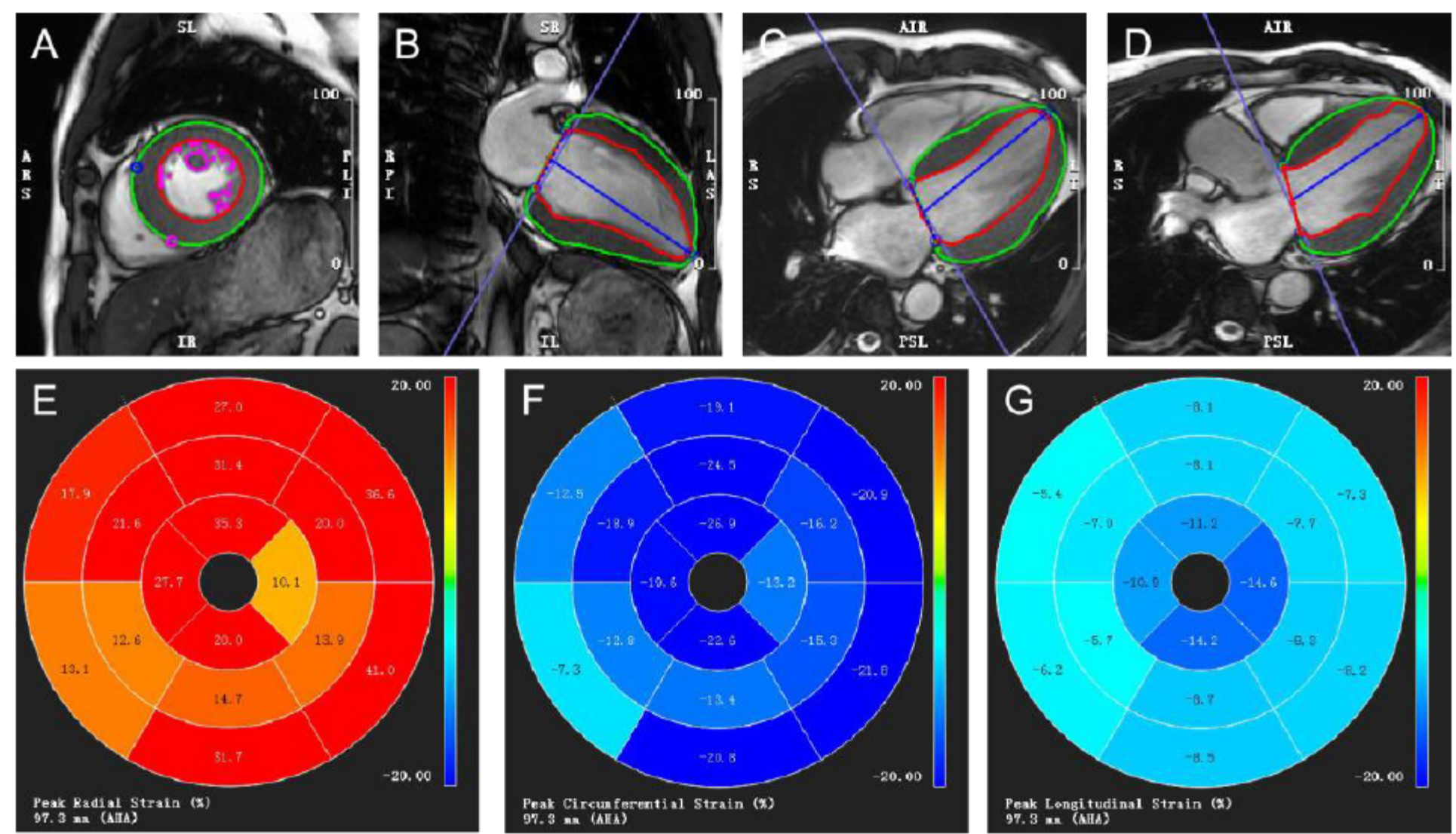

\section{Figure 3}

Examples of measuring LV myocardial strain by Tissue Tracking software. Standard cardiac images of short-axis (Plane A), two-chamber (Plane B), four-chamber (Plane C) and three-chamber views (Plane D); AHA segmentation of radial (Plane E), circumferential (Plane F) and longitudinal (Plane G) strain.
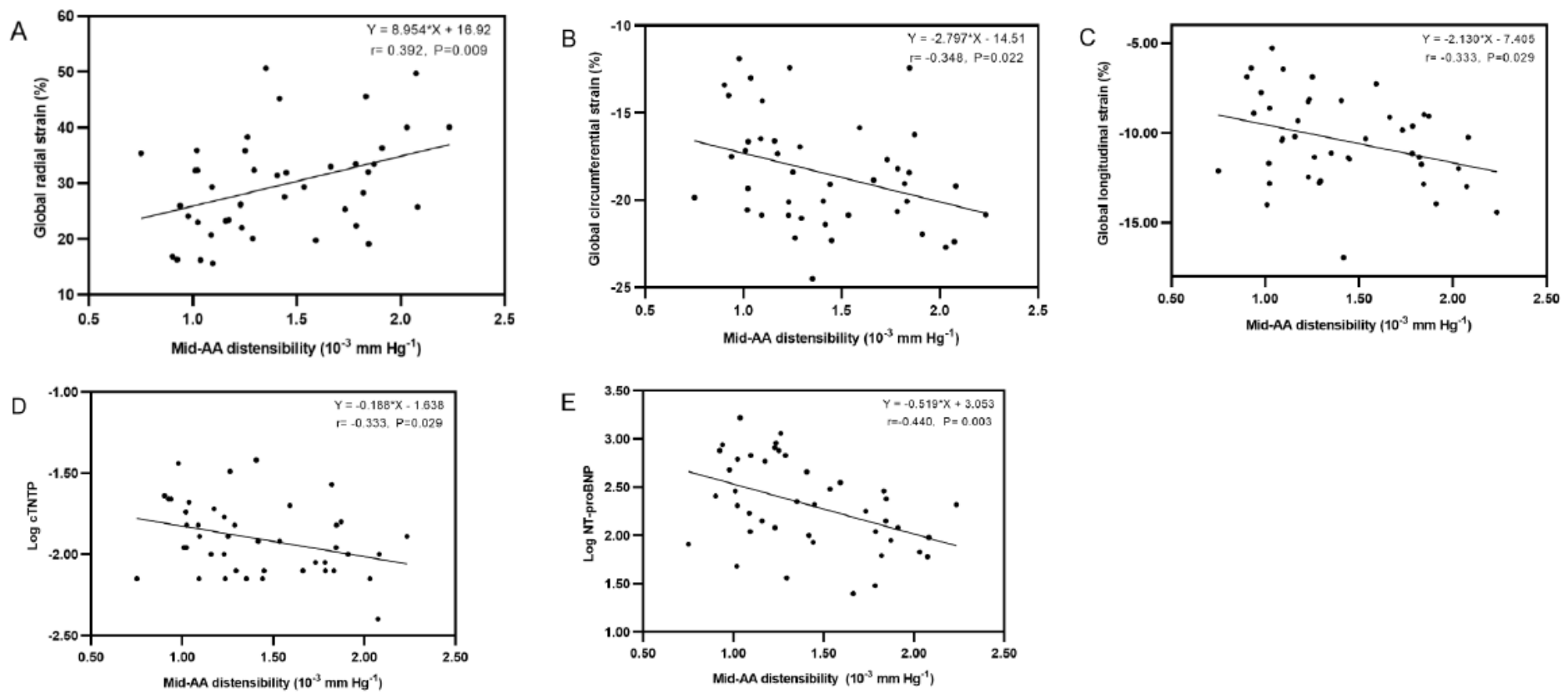

Figure 4 
Relationship between Mid-AA distensibility and global radial (Plane A), circumferential (Plane B) and longitudinal (Plane C) strain, cTNT (Plane D), NT-proBNP (Plane E) in BAV patients. Mid-AA middle ascending aorta, cTNT cardiac troponin T, NT-proBNP N-terminal pro-B type natriuretic peptide. 